\title{
3D models related to the publication: Morphology of the human embryonic brain and ventricles
}

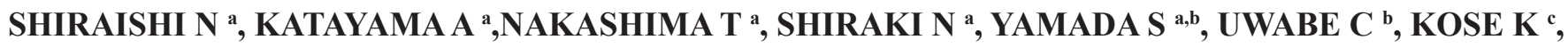 \\ TAKAKUWA T ${ }^{\text {a* }}$
}

${ }^{a}$ Human Health Science, Graduate School of Medicine, Kyoto University, Kyoto, Japan, 606-8507

${ }^{b}$ Congenital Anomaly Research Center, Graduate School of Medicine, Kyoto University, Kyoto, Japan, 606-8501

c Institute of Applied Physics, University of Tsukuba, Ibaragi, Japan, 305-8573

* corresponding author: Dr. Tetsuya Takakuwa(tez@hs.med.kyoto-u.ac.jp)

Abstract: The present 3D Dataset contains the 3D models analyzed in : Shiraishi N et al. Morphology and morphometry of the human embryonic brain: A three-dimensional analysis, NeuroImage, 115, 2015, 96-103, DOI: 10.1016/j.neuroimage.2015.04.044.

Key words: human brain, human embryo, magnetic resonance imaging, three-dimensional reconstruction

Submitted 14.07.2015, Accepted 23.07.2015. doi: 10.18563/m3.1.3.e3

(C) Copyright Tetsuya Takakuwa 2015

\section{SPECIMEN LIST}

The morphogenesis of the cerebral ventricles was visualized using images derived from human embryo specimens between Carnegie stage (CS) 13 and 23 from the Kyoto Collection (Congenital Anomaly Research Center, Graduate School of Medicine, Kyoto University), which were acquired with a magnetic resonance microscope equipped with a 2.35 -T superconducting magnet.

\begin{tabular}{|c|l|l|}
\hline Specimen ids & Species & Developmental Stage \\
\hline M3\#24_KC-CS13BRN50455 & Homo sapiens & Carnegie stage 13 \\
\hline M3\#25_KC-CS14BRN18834 & Homo sapiens & Carnegie stage 14 \\
\hline M3\#26_KC-CS15BRN19975 & Homo sapiens & Carnegie stage 15 \\
\hline \hline M3\#27_KC-CS16BRN7870 & Homo sapiens & Carnegie stage 16 \\
\hline M3\#28_KC-CS17BRN26702 & Homo sapiens & Carnegie stage 17 \\
\hline M3\#29_KC-CS18BRN25914 & Homo sapiens & Carnegie stage 18 \\
\hline M3\#30_KC-CS19BRN16508 & Homo sapiens & Carnegie stage 19 \\
\hline M3\#31_KC-CS20BRN26581 & Homo sapiens & Carnegie stage 20 \\
\hline \hline M3\#32_KC-CS21BRN33434 & Homo sapiens & Carnegie stage 21 \\
\hline M3\#33_KC-CS22BRN27960 & Homo sapiens & Carnegie stage 22 \\
\hline M3\#34_KC-CS23BRN28189 & Homo sapiens & Carnegie stage 23 \\
\hline
\end{tabular}

\section{METHODS}

Well-preserved human embryos between CS 13 and CS 23 were selected from Kyoto Collection for MR microscopic imaging (Nishimura et al, 1968; Shiota et al, 2007; O'Rahilly and Müller, 1987). The MR images of the embryos were acquired using a super-parallel MR microscope developed with a $2.35 \mathrm{~T}$ horizontal bore $(40 \mathrm{~cm})$ superconducting magnet (Matsuda et al., 2007). The brain tissue and ventricles were segmented for 3D reconstruction using FSL View of FMRIB Software LibraryTM (ver. 4.1.9, Analysis Group, FMRIB, Oxford, UK). Three-dimensional morphology of the brain tissue and ventricles were computationally reconstructed with AmiraTM software (ver. 5.4.0, Visage Imaging, Berlin, Germany). The 3D surface models were then processed with ISE-MeshTools (Lebrun, 2014); each model was orientated, tagged and labelled using this software. All tagged surfaces are provided in .vtk format, and labels in .flg format. All 3D surface models are also provided in .ply format, and can therefore be opened with a wide range of freeware. Representative lateral view of the brain tissue and ventricles are shown in Figure 1.

This study was approved by The Committee of Medical Ethics of Kyoto University Graduate School of Medicine, Kyoto, Japan (E986). 


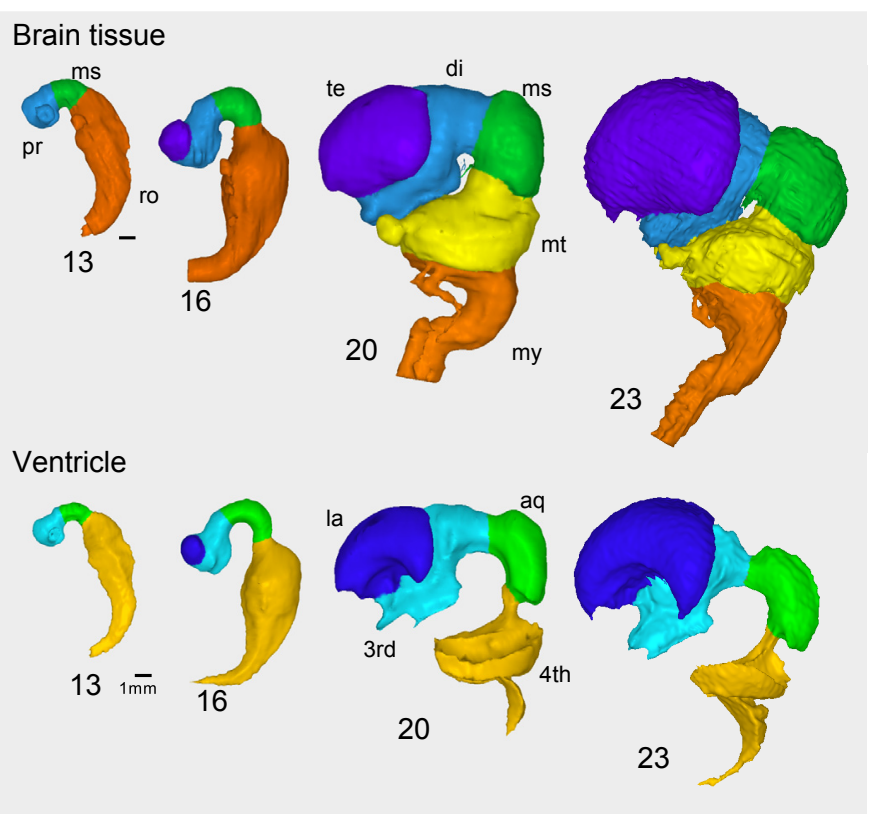

Figure 1: Representative lateral view of the brain tissue and ventricles. pr; prosencephalon, ms; mesencephalon, ro; rhombencephalon, te; telencephalon, di; diencephalon, mt; metencephalon, my; myelencephalon, la; lateral ventricle, $3 \mathrm{rd}$; third ventricle, aq; aqueduct of midbrain, 4th; fourth ventricle.

\section{ACKNOWLEDGEMENTS}

Grant sponsor: The Japan Society for the Promotion of Science; Grant number: \#24119002, \#25461642, \#26220004, \#15H01119, \#15K08134, \#15H05270, \#15H01121, \#15K15014: Japan Society for the Promotion of Science and BIRD of Japan Science and Technology Agency (JST).

\section{BIBLIOGRAPHY}

Lebrun, R., 2014. ISE-MeshTools, a 3D interactive fossil reconstruction freeware. 12th Annual Meeting of EAVP, Torino, Italy.

Matsuda, Y., Ono, S., Otake, Y., Handa, S., Kose, K., Haishi, T., Yamada, S., Uwabe, C., Shiota, K., 2007. Imaging of a large collection of human embryo using a superparallel MR microscope. Magn Reson Med Sci 6, 139146. DOI: $10.2463 / \mathrm{mrms} .6 .139$

Nishimura, H., Takano, K., Tanimura, T., Yasuda, M., 1968. Normal and abnormal development of human embryos: first report of the analysis of 1,213 intact embryos. Teratology 1, 281-290. DOI: 10.1002/tera.1420010306

O’Rahilly, R., Müller, F., 1987. Developmental stages in human embryos: including a revision of Streeter's Horizons and a survey of the Carnegie Collection. Washington, D.C.: Carnegie Institution of Washington.

Shiota, K., Yamada, S., Nakatsu-Komatsu, T., Uwabe, C., Kose, K., Matsuda, Y., Haishi, T., Mizuta, S., Matsuda, T., 2007. Visualization of human prenatal development by magnetic resonance imaging (MRI). Am J Med Genet A 143A, 3121-3126. DOI: 10.1002/ajmg.a.31994

Shiraishi N, Katayama A, Nakashima T, Yamada S, Uwabe C, Kose K, Takakuwa T, Morphology and morphometry of the human embryonic brain: A three-dimensional analysis, NeuroImage, 115, 2015, 96-103, DOI: 10.1016/j.neuroimage.2015.04.044 